\title{
Simulation of solid state lasers and amplifiers
}

\section{Christoph Pflaum, Zhabiz Rahimi}

Christoph Pflaum, Zhabiz Rahimi, "Simulation of solid state lasers and amplifiers," Proc. SPIE 9793, Education and Training in Optics and Photonics: ETOP 2015, 97930D (8 October 2015); doi: 10.1117/12.2223064

SPIE Event: Education and Training in Optics and Photonics: ETOP 2015, 2015, Bordeaux, France 


\title{
Simulation of Solid-State-Lasers and Amplifiers \\ Christoph Pflaum*a, Zhabiz Rahimi ${ }^{\mathrm{b}}$ \\ ${ }^{a}$ Friedrich Alexander-Universität Erlangen-Nürnberg; ${ }^{\text {} A S L D ~ G m b H, ~ G e r m a n y ~}$
}

\begin{abstract}
Modern simulation of solid state lasers and amplifiers is a multi-physics problem. It requires the simulation of light with different techniques, as well as coupling of optical effects with other physical effects like deformation and stress inside a laser crystal. We present and overview simulation techniques which are necessary for an accurate modelling of solid state laser. These include ray tracing for modeling of pump light, Gauss mode analysis for stability calculations of a laser cavity, and vectorial beam propagation method for the amplifier simulation. It is also necessary to perform mechanical or rate equations simulations. Structural mechanics simulation is required for the calculation of thermal lens and thermally induced birefringence effects inside a laser crystal. Coupling Gauss mode analysis and rate equations leads to the dynamic mode analysis (DMA), which is used to calculate output power and beam quality of a laser. Furthermore, we present a mode amplification method, which allows us to simulate spectral narrowing in laser amplifiers and power and beam quality calculation.

The above mentioned simulation techniques have been integrated into laser simulation software package ASLD. We have used highly efficient algorithms and modern software techniques, together with a user friendly GUI. This enables users to efficiently simulate complex resonator and amplifier designs and save their development time and costs. Furthermore, ASLD can be used for educational purpose at universities and research institutes.
\end{abstract}

Keywords: Laser simulation, thermal lensing, pulse amplification

\section{INTRODUCTION}

Laser is a fascinating technology with a wide range of applications. There are many different physical effects associated with lasers, for example properties like high intensity beam power in case of ultra-short pulses. These effects make both studying of laser physics and optimization of lasers a difficult task. Therefore, a laser simulation program is a helpful tool to look inside a laser and analyze the properties of a specific laser configuration. For solid state lasers, we developed laser simulation program ASLD, which meets the requirements for both educational and industrial applications. It also allows us to model and simulate different physical effects, which are important for an accurate laser simulation. These physical effects are (see [4]):

- thermal lens effect including a detailed thermal and mechanical analysis of a laser crystal

- gain and dynamic behavior of the laser light, including spectral and temperature dependence of all physical parameters

- $\quad$ stability of the resonator cavity and influence of optical elements on beam radius

- birefringence inside a laser crystal and impact on depolarization of the beam and losses

- $\quad$ spectral analysis including stretching and compressing of a short laser pulse

- $\quad$ analysis of the pump light configuration

Several simulation techniques have to be applied in order to simulate these different physical effects. Furthermore, a suitable software concept is required to couple these simulation techniques in a software package. ASLD is designed and implemented using a software concept designed for this specific application. Each software module has its input and output parameters, which can be specified and modified by the user. A change in these parameters causes only affected modules to be recalculated. Figure ... depicts the main modules of ASLD and their relations. Let explain simulation techniques of these modules in more detail.

*Christoph.pflaum@ fau.de; phone +49 - (0)9131 - 85 - 28692; www.asldweb.com/

Education and Training in Optics and Photonics: ETOP 2015, edited by Eric Cormier, Laurent Sarger Proc. of SPIE Vol. 9793, 97930D · @ 2015 SPIE, IEEE, OSA, ICO · doi: 10.1117/12.2223064 


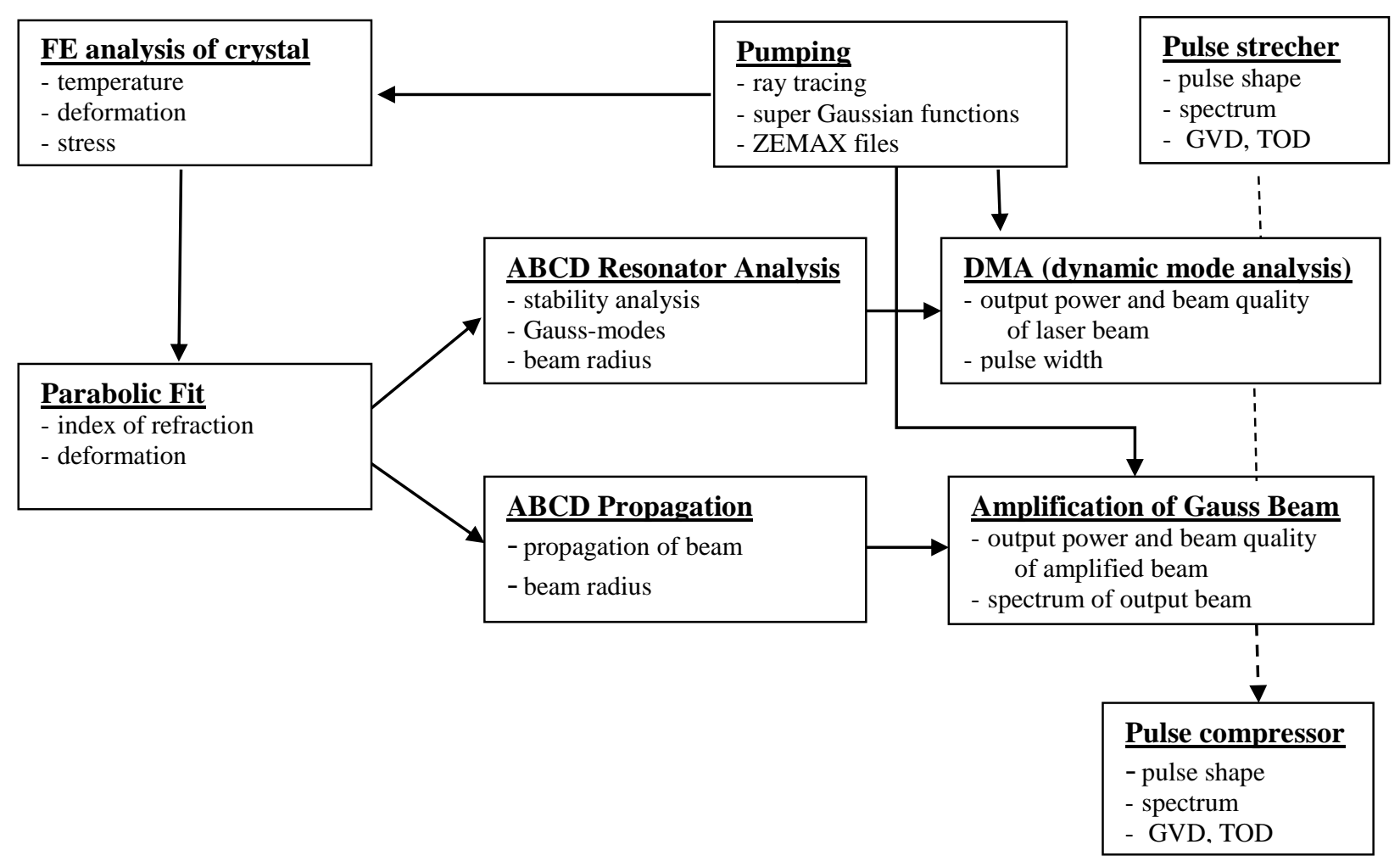

\section{SIMULATION TECHNIQUES}

\section{Modelling of pump light}

Depending on properties of the pump light, different techniques have to be applied to simulate the pump light. Diode light is usually a multimode light. Then, ray tracing is an optimal simulation technique to calculate the absorbed pump light distribution. However, if the pump light is a laser beam, super Gaussian functions are more appropriate for describing pump light propagation inside a laser crystal. It is important is to take into account the crystal's doping concentration. By bonding crystals with different doping concentrations, we can obtain a crystal with different doping concentrations at its different sections (see [5]). Figure 1-a) shows the simulated pump light absorption for such an endpumped laser rod.

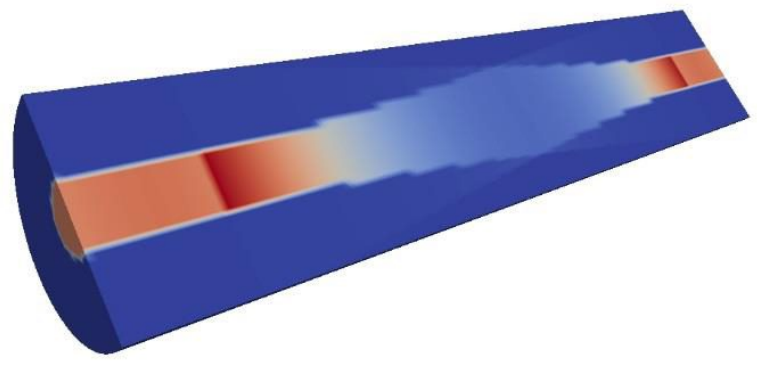

a)

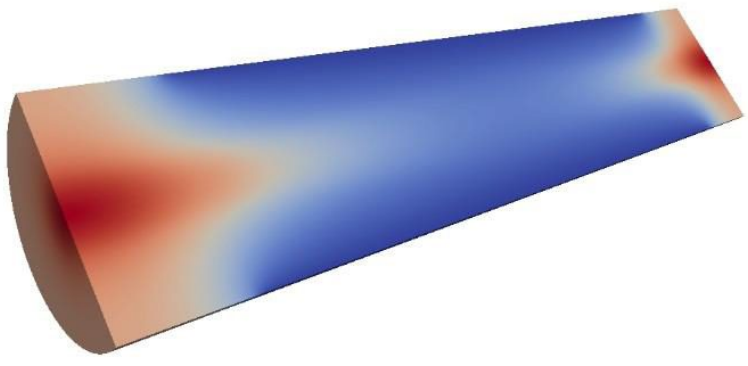

b)

Figure 1. Nd:YAG crystal with doping concentration of $0.3 \%, 0.4 \%$, and $0.3 \%$ at different sections. a) Absorbed pump power in laser crystal, b) Resulting temperature distribution. 


\section{Finite element analysis of temperature and deformation}

Finite element is a well-known method for the calculation of temperature and deformation caused by pumping a crystal. The thermal boundary conditions have to be specified accurately. Figure 1-b) shows the temperature distribution calculated for an end-pumped laser rod, where the crystal was cooled only at an interior section. The resulting "von Mises" stress is illustrated in Figure 2-a). Using the stress inside the crystal, one can calculate the stress induced birefringence, which influences the depolarization of beam (see Figure 3).

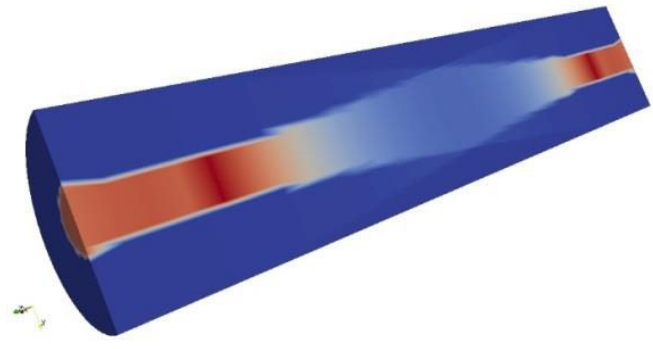

a)

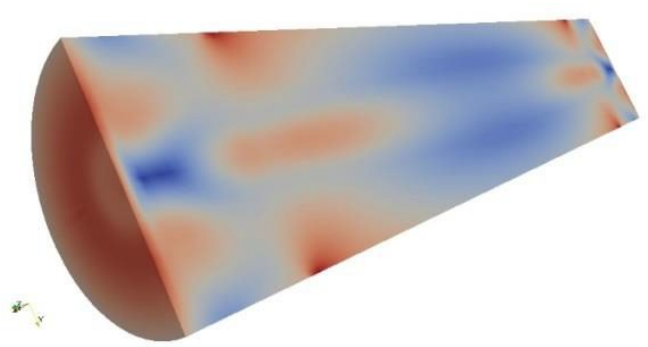

b)

Figure 2. Nd:YAG crystal with doping of $0.3 \%, 0.4 \%$, and $0.3 \%$ atn different sections. a) Population inversion b) Von Mises stress.

\section{Gauss mode analysis}

Gauss mode analysis applies ABCD matrices for each optical element inside a laser resonator. The ABCD matrix of the laser crystal is obtained by a product of several $A B C D$ matrices corresponding to end faces of the crystal and a sequence of Gaussian ducts inside the crystal. To this end, the deformation of the end faces and the refractive index inside the crystal has to be fitted by parabolic functions. The results of a Gauss mode analysis are stability information of the resonator and the beam radius inside cavity for stable resonators. Beam radius is needed to calculate overlap integrals for the dynamic mode analysis.

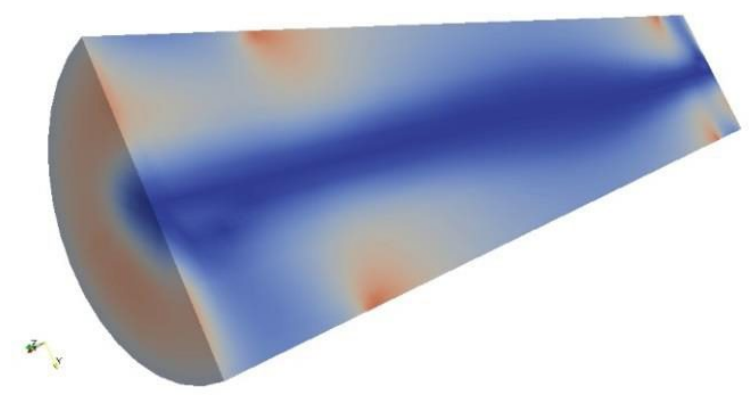

a)

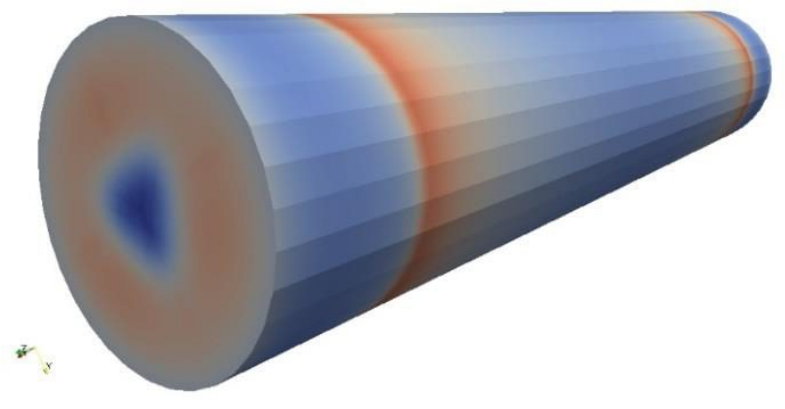

b)

Figure 3. Nd:YAG crystal with doping $0.3 \%, 0.4 \%$, and $0.3 \%$ in different sections. Birefringence for 111-cut.

\section{Dynamic Mode Analysis DMA}

The dynamic mode analysis is a highly efficient simulation technique that calculates output power and beam quality of a laser resonator. It applies a finite volume discretization for the population inversion (see [6]). Figure 2-b) depicts the calculated 3-dimensional population inversion obtained for the pump configuration in Figure 1 a). Using these 3dimensional simulation results, one can modify pump configuration and beam radius to obtain an optimal overlap of both. By this approach, one can optimize output power and beam quality of the laser resonator. The numerical algorithm of DMA solves a system of rate equations for population inversion and high and low orders modes. This system is solved by a highly efficient adaptive time stepping method. The dynamic mode analysis can be applied for 3-level and 4-level 
laser systems. Parameters like stimulated emission cross section and reabsorption cross section can be modeled temperature-, wavelength- and space-dependent, since population inversion is discretized on a finite volume mesh. This leads to more accurate simulation results, than taking a spatial average of these parameters. ASLD applies dynamic mode analysis for passive and active Q-switches, passively Q-switched SESAM, intra-cavity frequency doubling and regenerative amplifiers.

\section{Amplifier simulation using Gaussian functions}

There are different simulation techniques to calculate the gain of a laser amplifier. Similar to resonators it is important to consider 3-dimensional behavior of the pumping configuration and population inversion. Then, one can either apply a beam propagation method or restrict himself to the gain of high and low order Gaussian functions. This second approach is less computationally intensive and gives clear information how beam quality changes through the amplification of laser beam. The input beam can be chosen to be the output of a laser resonator. There are two approaches to include polarization effects. One is to calculate the depolarization of single rays through the amplifier and combine this with the amplification of each Gaussian functions. The other approach is the vectorial beam propagation method, which is more accurate but also more computationally intensive.

\section{Vectorial Beam Propagation Method}

The vectorial beam propagation method applies a paraxial approximation of the vector Helmholtz equation obtained by Maxwell's equations (see [1]). It is discretizes the electrical field vector $\left(E_{x}, E_{y}\right)$ in $x y$-plane and propagates it step by step in propagation direction (z-direction). Using this approach, the birefringence effects, like in Figure $3 b$ ) can be accurately calculated (see [2]).

\section{Simulation of ultrashort pulses}

ASLD can simulate chirped pulse amplification of short pulses. Due to the high energy of the pulse, it has to be stretched before amplification in order to avoid damage to the laser system. The pulse is re-compressed after amplification. Both stretcher and compressors consist of mirrors and gratings. Simulations take into account that the spectrum of the pulse might be narrowed by limited gain bandwidth (see [3]).

\section{CONCLUSION}

ASLD is a multi-functional software tool and can be used for educational purpose, research institutes as well as industries. It assists developers and engineers to build lasers with less number of expensive try and error experiments. It is also helpful for students who want to analyze and understand various effects associated with laser beam generation and propagation inside a cavity and amplifier.

\section{REFERENCES}

[1] Graupeter, T., Hartmann, R., Pflaum, C. "Calculations of Eigenpolarization in Nd:YAG Laser Rods Due to Thermally Induced Birefringence”. IEEE Journal of Quantum Electronics, 50 (12), 1035 - 1043, (2014).

[2] Hartmann R., Pflaum C., Graupeter T., "Analysis of thermal depolarization compensation using full vectorial beam propagation method in laser amplifiers", Proc. SPIE 9343, (2015).

[3] Huabao Cao, Xingqiang Lu, Dianyuan Fan, "Numerical simulation of gain narrowing control by hybrid amplifiers chain based on Ti:sapphire and Ti:chrysoberyl”, Optics Communications, 284 (6), 1622-1625, (2011).

[4] Koechner, W., [Solid-state laser engineering], Springer, Berlin, (2006).

[5] Rahimi, Z., Pflaum, C., "Simulation of solid-state lasers with composite and ceramic crystals", Proc. SPIE 8599, (2013).

[6] Wohlmuth, Matthias; Pflaum, Christoph; Hahn, C., "Dynamic multimode analysis of Q-switched solid state laser cavities", Optics Express, 17, 17303-17316 (2009). 